\title{
A nanofluidic device for rapid and multiplexed SARS-CoV-2 serological antibody detection
}

\section{Thomas Mortelmans}

Paul Scherrer Institute

\section{Dimitrios Kazazis}

Paul Scherrer Institute

Celestino Padeste

Paul Scherrer Institute

\section{Philipp Berger}

Paul Scherrer Institute

\section{Xiaodan Li}

Paul Scherrer Institute

Yasin Ekinci ( $\nabla$ yasin.ekinci@psi.ch )

Paul Scherrer Institute

\section{Research Article}

Keywords:

Posted Date: May 18th, 2021

DOI: https://doi.org/10.21203/rs.3.rs-537056/v1

License: (c) (i) This work is licensed under a Creative Commons Attribution 4.0 International License.

Read Full License 


\section{Abstract}

The outbreak of COVID-19 has led to a substantial death toll and has hindered the functioning of modern society, sending the world into a medical and economic crisis1,2. This underlined the importance of pointof-care diagnostics, as well as accurate, cost-effective serological antibody tests as well as point-of-care diagnostics to monitor the viral spread and contain pandemics and endemics. Here, we present a threedimensional (3D) nanofluidic device for rapid and multiplexed detection of viral antibodies. The device is designed to size-dependently immobilize particles from a multi-particle mixture at predefined positions in nanochannels through capillary forces only, resulting in distinct trapping lines. We show that individual lines can be used as an on-chip fluorescence-linked immunosorbent assay for multiplexed detection of serological immunoglobulin antibodies against viral proteins with high sensitivity. Further device versatility is exhibited by on-bead color multiplexing for simultaneous detection of IgG and IgM antibodies in convalescent human serum and by concurrent detection of anti-spike (SARS-CoV-2) and anti-hemagglutinin (Influenza A) antibodies. The device's applications can be further extended to detect a plethora of diseases simultaneously in a reliable and straightforward manner.

\section{Main Text}

Currently, SARS-CoV-2 diagnostic testing is primarily performed in a clinical setting, where either a nasal swab or blood sample is taken by a clinician and sent for further testing in a biosafety level 2 facility. Here, trained medical personnel characterizes the biospecimen mostly with respect to either SARS-CoV-2 related genetic material or associated antibodies with a PCR or ELISA test respectively ${ }^{3}$. In light of these highly reliable yet cumbersome methods, there is a clear benefit for devices and methods that can provide this information rapidly and cost-effectively without the need for specialized laboratories. Innovative testing solutions are even more needed taking into account that $30 \%$ of all SARS-CoV- 2 infections are asymptomatic ${ }^{4}$. Additionally, the previously commercialized lateral flow assays (LFAs), despite their speed and cost advantages, have a limited sensitivity and reproducibility and only provide binary information (yes/no) on a possible previous infection ${ }^{5-7}$.

In this letter, we first introduce a 3D poly(methyl methacrylate) (PMMA) nanofluidic particle sorter fabricated by state-of-the-art nanofabrication methods. We, then, demonstrate the potential of the device to perform on-chip multiplexed serological assays for anti-spike (SARS-CoV-2) IgG while providing semiquantitative data with a high degree of sensitivity and specificity. The proposed device is based on fluorescence measurements of trapped biofunctionalized nanoparticles, allowing for high sensitivity as well as point-of-care use ${ }^{8}$, in contrast to other on-bead or microfluidic methods ${ }^{9,10}$. The device's versatility and performance are shown by simultaneous on-bead testing of different antibody sub-types as well as concurrent detection of SARS-CoV-2 and Influenza A antibodies. As both infections provoke similar symptoms ${ }^{11}$, this kind of differential testing would allow the treating physician to provide the correct diagnosis and treatment rapidly and accurately. 
Our nanofluidic device is designed to be low-cost and easy to operate. It is entirely made of PMMA and contains only passive capillary microfluidic elements to control the fluid flow without the need for external loading mechanisms, such as syringes or pressure pumps. The critical feature of the device is a wedge that enables geometrical trapping of nanoparticles (Fig. 1a and j) at a predetermined location, allowing for particle size sorting and size determination (Table S1).

The chip's inflow region consists of three parallel channels with separate inlets (three channels in the present case) to enable redundancy in testing or multiplexing (Fig. 1f). The applied liquid is passively aspirated into the 300- $\mu \mathrm{m}$-wide inflow, enhanced pillars with a $20 \mu \mathrm{m}$ diameter and $40 \mu \mathrm{m}$ pitch (Fig. 1c). The channels containing various passive flow elements were optimized for a controlled and reproducible flow. More specifically, the size and pitch of the incorporated pillars were altered throughout the device (Fig. 1c-e) to modulate fluid flow rate and to provide necessary support during the bonding of these relatively broad, but extremely shallow channels (aspect ratio 375:1) (Fig. S.2). Furthermore, to minimize premature drying of the liquid inside the nanofluidic device and to ensure a constant fluid filling velocity, an inflow resistor was incorporated (Fig. 1b). This was achieved by narrowing the channel width after the inflow region to $100 \mu \mathrm{m}$ and keeping it constant for a length of $500 \mu \mathrm{m}$. Afterward, the width is gradually increased to $300 \mu \mathrm{m}$ (Fig. $1 \mathrm{~b}-$ I.R.). The IR is followed by the trapping region (TR), i.e. a wedge-structure with a linearly decreasing the channel height from 3.4 to $0.8 \mu \mathrm{m}$ over a distance of $1.2 \mathrm{~mm}$.

After the TR, the channel height is kept constant at $0.8 \mu \mathrm{m}$ for $100 \mu \mathrm{m}$ before a secondary wedge increases the total height to $1 \mu \mathrm{m}$ over $1 \mathrm{~mm}$. This connecting wedge (CW) couples the active device region to a capillary pump containing $20 \mu \mathrm{m}$ pillars with a $30 \mu \mathrm{m}$ pitch (Fig. 1e). The TR and CW necessitate nanoscale topography control over several millimeters and simultaneous integration of various micro and nanofluidic components. To overcome these challenging fabrication requirements, high precision gray scale e-beam lithography ( $g-E B L)$ is employed to pattern the nanofluidic device following a process that we developed recently through extensive process optimization ${ }^{12}$. This highresolution structure is subsequently replica-molded to obtain a negative polymeric stamp, enabling costeffective upscaling of fabrication through nanoimprint lithography (Fig. S1).

The topography variation in the TR is linear and patterned with high accuracy (Fig. 1g-h). The interference colors (Fig. $1 \mathrm{~h}$ - inset) visible after bonding evidence the preservation of the 3D profile inside the nanofluidic channel. Before proceeding with the particle trapping experiments, the devices were functionalized with poly(vinyl alcohol) (PVA). The application procedure of PVA was shown to influence the flow behavior of the particles in the nanofluidic channels and was extensively optimized to maintain a high flowrate during the entire particle trapping experiment (Sup. Video 1). The functionalization blocks non-specific binding sites on the PMMA ${ }^{13}$ (Fig. S3) and allows for more controlled surface wetting ${ }^{14}$. The latter is evidenced by the absence of corner flow in the capillary pump region and improvement of the filling front (Fig 1i; Fig. S4; Sup. Video 2). The device concept with various micro and nanofluidic components in combination with specific surface chemistry allows for facile device operation that can be used in various applications. This includes particle size determination, sorting, and immobilization, as well as paving the way for applications such as in point-of-care diagnostics. 
The ease-of-use and the performance of the device was highlighted by passive size-dependent particle immobilization of five different fluorescent polystyrene calibration grade particles in the TR (Fig. $1 \mathrm{j}$-k). The relationship between the particle trapping position and its nominal diameter was shown to be highly linear $\left(R^{2}=0.998\right.$; Fig. 11$)$ and could be used to effortlessly determine particle sizes with nanometer accuracy (Table S1). Extrapolation from the linear fit (Fig. 1j), amounts to a minimal TR channel height of $720 \mathrm{~nm}$, indicating a reduction of roughly $45 \mathrm{~nm}$ when compared to the unbonded height of $765 \mathrm{~nm}$. This can be attributed to the surface selective bonding process ${ }^{15-17}$ and also highlights the necessity of supporting pillars inside the nanofluidic channel (Fig. 1h, inset; Fig. S2).

By using the size-dependent immobilization properties of our nanofluidic device, we developed an onbead immunoassay for the detection of specific SARS-CoV-2 antibodies in human serum by concentrating the relevant nanoparticles at predetermined locations. Our immune system fights infection and eliminates foreign bodies using innate and adaptive mechanisms, where the latter requires growth and rearrangement of gene elements to produce antibodies that specifically bind to invading antigens. The most commonly found antibody type in human serum is immunoglobulin $\mathrm{G}(\lg \mathrm{G})^{18}$, making it a valuable target for immune status characterization. One of the major antigens of SARS-CoV-2 associated IgG antibodies is the surface trimeric spike (S) protein, which plays a vital role in facilitating the entry of the SARS-CoV-2 into human cells ${ }^{19}$. The receptor-binding domain (RBD) of the S-protein (S-RBD) is an immunodominant target for SARS-CoV-2 antibodies ${ }^{20}$. To enable their on-chip detection, we used the high-affinity interaction between streptavidin and biotin to couple biotinylated S-RBD to the surface of 2.8 $\mu \mathrm{m}$ streptavidin-coated beads (Fig. 2b; top).

Once functionalized, the beads were mixed with human serum. If the patient was previously exposed to SARS-CoV-2, the serum will contain antibodies with a specific antigen-binding site for S-RBD and bind to the beads. The latter can be fluorescently visualized by conventional immunostaining procedures. In our case, a donkey-originating antibody conjugated with a red fluorescent dye (Cy5) was used that specifically binds to human IgG (Fig. 2a; left). To benchmark the serological immunoassay, the lowest detectable concentration of a purified humanized anti-S-RBD IgG antibody in human serum was determined. To evaluate the nanofluidic device function, non-functionalized $0.9 \mu \mathrm{m}$ calibration-grade particles were added to the suspension, acting as a flow control before applying the particle mixture onto the device's inflow region. Analysis of the corresponding fluorescent signal of the trapped beads revealed a limit-of-detection (LOD) around $1 \mathrm{nM}$ (Fig. 2c). This sensitivity is comparable to the state-of-the-art microfluidic devices ${ }^{10,21,22}$, whereas the present device is more straightforward to use and has a low infrastructure requirement. Furthermore, in recent studies, the physiologically relevant concentration of this specific type of anti-spike IgG antibody in recovered COVID-19 infected patients has been shown to be in the range of $9.6-28880 \mathrm{nM}^{23}$. This shows that the developed on-bead and on-chip nanofluidic device operates well within the physiologically relevant concentrations.

Additionally, the assay was validated using a patient serum set containing both PCR positive $(n=19)$ and PCR negative $(n=10)$ patients (Fig. 2 d). From the 29 different samples, 28 could be identified correctly 
according to infection status, with one false negative result. The signal distribution of positive cases is to be expected, as immune responses are highly individual and dependent on the infection history of the patient. This corresponds to a test sensitivity of $94.7 \%$ (99-65) with a specificity of $100 \%(100-72)$ and an area under the curve of $0.95\left(\sigma_{\overline{\mathrm{x}}}=0.05\right)$ according to receiver operator characteristic (ROC) analysis. We note that the false negative was retested and showed a signal above the threshold value. Although we could not reconstruct the root cause of the initial false negative during the blind experimental run, the overall results show the performance of the devices in this early development phase and highlights the potential for further optimization. These findings evidence the applicability of the assay in a real-world setting for the IgG serological characterization of suspected COVID-19 patients ${ }^{24}$.

Even though IgG is the dominant immunoglobulin subtype in human serum, it is mainly associated with late-stage and memory-related immune responses. In the case of SARS-CoV-2 infection, specific IgG antibodies become prominent within 7 days post-infection. This hampers the applicability of IgG-specific antigen assays for early-stage disease detection. However, other antibody sub-types, such as immunoglobulin $\mathrm{M}(\mathrm{IgM})$, can be already found in human serum 4 days post infection ${ }^{25}$. Hence, the onbead and on-chip assay was further developed to include color-multiplexing for simultaneous detection of both antibody subtypes (Fig. 2e; Fig. S5). More specifically, the immunostaining solution contained anti-human IgM antibodies conjugated with a green fluorescent dye (Alexa 488) as well as anti-human IgG antibodies conjugated with a red fluorescent dye (Cy5). The anti-IgG fluorescent signal of the COVID19 positive patient was 25.5 times greater than that of the negative control. Similarly, the anti-IgM signal was 4.9 times higher (Fig. 2f). This difference between IgG and IgM is to be expected, given that the investigated COVID-19 positive serum was taken 33 days after symptom onset. As is well known, IgM antibody levels start to decline roughly 21 days after infection, explaining the findings ${ }^{26}$. This experiment shows the potential of color multiplexing to monitor the IgG and IgM response of suspected SARS-CoV-2 infected people.

Furthermore, it is of interest to perform simultaneous differential testing for the presence of antibodies against diseases with similar symptoms. In the case of COVID-19, one of the most symptomatically similar and prevalent viral infections is Influenza A. The latter causes yearly epidemics and it is one of the major targets of annual vaccination campaigns ${ }^{27}$. To demonstrate the on-chip multiplexing of diseasespecific antibodies, 1- $\mu \mathrm{m}$-size streptavidin-coated beads were labeled with biotinylated hemagglutinin, i.e., immunodominant influenza A-associated protein (Fig 2b; bottom) ${ }^{28}$. The surfaces of the $1 \mu \mathrm{m}$ and 2.8 $\mu \mathrm{m}$ particles biofunctionalized with hemagglutinin and S-RBD, respectively, were saturated with free biotin before being added together. This successfully inhibited the aggregation of the SARS-CoV-2 and Influenza A functionalized particles (Fig. S5) and enabled size separation in the 3D nanofluidic device. As a proof-of-principle experiment, the multi-particle suspension was mixed with various combinations of purified polyclonal rabbit antibodies, targeting either the S-RBD or hemagglutinin protein (Fig $2 \mathrm{~g}$ ). The beads were subsequently immunostained with a Cy3 anti-rabbit antibody. The obtained signal at their trapping positions is shown to correlate very well with the presence or absence of the relevant antibodies for either S-RBD (SARS-CoV-2) or hemagglutinin (Influenza A), respectively (Fig. 2h). This proof-of- 
principle experiment further emphasizes the versatility of our 3D nanofluidic device within the framework of serological multiplexed immunoassays.

In conclusion, we have introduced a novel approach towards multiplexed antibody and disease testing by using a novel 3D PMMA-based nanofluidic device. Proof of principle was obtained by showing that calibration-grade particles can be size-dependently immobilized, and their size can be accurately determined from their trapping position. The size sorting capability of the device was used to concentrate and trap S-RBD-functionalized beads in an IgG SARS-CoV-2 serological assay with a detection limit well within the range of state-of-the-art immunoassays. The test was further cross-validated with PCR-tested patient samples, showing a high degree of sensitivity and specificity. Moreover, on-bead color multiplexing has demonstrated the potential to simultaneously monitor the presence of both $\lg \mathrm{g}$ and $\operatorname{lgM}$ antibodies in human sera on single particles for future time-dependent antibody studies. Additionally, we have highlighted the versatility and applicability of the 3D nanofluidic device by detecting anti-S-RBD (SARS-CoV-2) and anti-hemagglutinin (Influenza A) antibodies in control samples using different bead sizes.

Here we showed multiplexing in two-dimensions, by varying particle size and the conjugated dye of the detection antibody. For future work, we aim to extend this by using various fluidic channels for different immunoassays and using color-coded beads of a similar size ${ }^{29}$. We believe that by this multiplexing in four dimensions, concurrent detection of more than 100 antibodies on a single-chip can be achieved. Moreover, we aim to transfer the fluorescence detection to a portable and compact microscope and eventually adaptable to smartphones, since the devices do not necessitate state-of-the-art infrastructure for immunoassay read-out. We note that the facile operation and cost-effective nature of the developed 3D nanofluidic device is not limited to on-chip serological immunoassays. Its applications could be further extended to various disciplines of biomedical sciences to address key research questions, ranging from mitochondrial size determination in Parkinson's disease ${ }^{30}$ to nanoparticle-based cancer therapeutics ${ }^{31}$, sickle-cell diagnosis ${ }^{32}$ and many more.

\section{Declarations}

\section{Acknowledgements}

T.M. is supported by the Swiss Nanoscience Institute, Project Number 1702. The authors gratefully thank Thomas Braun, Henning Stahlberg, Gabriel Aeppli, Helmut Schift and G.V. Shivashankar for the insightful discussions.

\section{Author contributions}

T.M., Y.E., and X.L. conceived and designed the experiments. T.M. and Y.E. designed the devices. D.K. assisted with the nanofabrication and acquired the SEM-images of the devices. T. M. optimized the device fabrication and immunoassays and performed all the experiments and data analysis. P.B. set up the fluorescence microscopy and validated the image analysis. C.P. assisted with and advised on the 
surface functionalization and device materials. Y.E. and X.L. supervised the project. All authors contributed to the writing of the manuscript.

\section{Competing interest statement}

The authors declare the following competing interests: EP21171944.

\section{References}

1. Zhou, P. et al. A pneumonia outbreak associated with a new coronavirus of probable bat origin. Nature 579, 270-273 (2020).

2. Gorbalenya, A. E. et al. The species Severe acute respiratory syndrome-related coronavirus: classifying 2019-nCoV and naming it SARS-CoV-2. Nat. Microbiol. 5, 536-544 (2020).

3. Marc Lipsitch, R. K. and M. J. M. Antibody testing will enhance the power and accuracy of COVID-19prevention trials. Nat. Med. 26, 814-817 (2020).

4. Nishiura, H. et al. Estimation of the asymptomatic ratio of novel coronavirus infections (COVID-19). Int. J. Infect. Dis. 94, 154-155 (2020).

5. Guglielmi, G. Rapid coronavirus tests: a guide for the perplexed. Nature 590, 202-205 (2021).

6. Rubin, R. The Challenges of Expanding Rapid Tests to Curb COVID-19. JAMA - J. Am. Med. Assoc. 324, 1813-1815 (2020).

7. Corman, V. M. et al. Comparison of seven commercial SARS-CoV-2 rapid point-of-care antigen tests: a single-centre laboratory evaluation study. 5247, 1-9 (2021).

8. Ung, Y. U. S., Ampa, F. E. C. \& Hih, W. E. I. H. S. Open-source do-it-yourself multi-color fluorescence smartphone microscopy. Biomed. Opt. Express 8, 236-247 (2017).

9. Mariën, J. et al. Evaluating SARS-CoV-2 spike and nucleocapsid proteins as targets for antibody detection in severe and mild COVID-19 cases using a Luminex bead-based assay. J. Virol. Methods 288, (2021).

10. Swank, Z. et al. A high-throughput microfluidic nanoimmunoassay for detecting anti-SARS-CoV-2 antibodies in serum or. PNAS 118, (2021).

11. Bhatraju, P. et al. Covid-19 in Critically III Patients in the Seattle Region - Case Series. N. Engl. J. Med. 2012-2022 (2020). doi:10.1056/NEJMoa2004500

12. Mortelmans, T. et al. Grayscale e-beam lithography: Effects of a delayed development for wellcontrolled 3D patterning. Microelectron. Eng. 225, 111272 (2020).

13. Rodda, D. J. \& Yamazaki, H. Poly(vinyl alcohol) as a blocking agent in enzyme immunoassays. Immunol. Invest. 23, 421-428 (1994).

14. Khodayari Bavil, A. \& Kim, J. A capillary flow-driven microfluidic system for microparticle-labeled immunoassays. Analyst 143, 3335-3342 (2018). 
15. Schleunitz, A. et al. Novel 3D micro- and nanofabrication method using thermally activated selective topography equilibration (TASTE) of polymers. Nano Converg. 1, 7 (2014).

16. Mironov, A. E. et al. Photolithography in the vacuum ultraviolet $(172 \mathrm{~nm})$ with sub- $400 \mathrm{~nm}$ resolution: Photoablative patterning of nanostructures and optical components in bulk polymers and thin films on semiconductors. Nanoscale 12, 16796-16804 (2020).

17. Bhattacharyya, A. \& Klapperich, C. M. Mechanical and chemical analysis of plasma and ultravioletozone surface treatments for thermal bonding of polymeric microfluidic devices. Lab Chip 7, 876882 (2007).

18. Chaplin, D. D. Overview of the immune response. J. Allergy Clin. Immunol. 125, S3-S23 (2010).

19. Chi, X. et al. A neutralizing human antibody binds to the N-terminal domain of the Spike protein of SARS-CoV-2. Science (80-. ). 369, 650-655 (2020).

20. Premkumar, L. et al. The receptor-binding domain of the viral spike protein is an immunodominant and highly specific target of antibodies in SARS-CoV-2 patients. Sci. Immunol. 5, 1-10 (2020).

21. Rodriguez-moncayo, R. et al. A high-throughput multiplexed microfluidic device for COVID-19 serology assays. Lab Chip 21, 93-104 (2021).

22. Hartanto, H., Wu, M., Lam, M. L. \& Chen, T. H. Microfluidic immunoassay for detection of serological antibodies: A potential tool for rapid evaluation of immunity against SARS-CoV-2. Biomicrofluidics 14, (2020).

23. Tan, X. et al. Rapid and quantitative detection of SARS-CoV-2 specific IgG for convalescent serum evaluation. Biosens. Bioelectron. (2020).

24. West, C. P., Montori, V. M. \& Sampathkumar, P. COVID-19 Testing: The Threat of False-Negative Results. Mayo Clin. Proc. 95, 1127-1129 (2020).

25. Liu, X. et al. Patterns of IgG and IgM antibody response in COVID-19 patients. Emerg. Microbes Infect. 9, 1269-1274 (2020).

26. Long, Q. X. et al. Antibody responses to SARS-CoV-2 in patients with COVID-19. Nat. Med. 26, 845848 (2020).

27. Solomon, D. A., Sherman, A. C. \& Kanjilal, S. Influenza in the COVID-19 Era. JAMA Insights 324, 2020-2021 (2020).

28. Dilillo, D. J., Tan, G. S., Palese, P. \& Ravetch, J. V. Broadly neutralizing hemagglutinin stalk-specific antibodies require FcR interactions for protection against influenza virus in vivo. Nat. Med. 20, 143151 (2014).

29. Germeraad, E. et al. The development of a multiplex serological assay for avian influenza based on Luminex technology. Methods 158, 54-60 (2019).

30. Bose, A. \& Beal, M. F. Mitochondrial dysfunction in Parkinson's disease. J. Neurochem. 139, 216-231 (2016).

31. Davis, M. E., Chen, Z. G. \& Shin, D. M. Nanoparticle therapeutics: an emerging treatment modality for cancer. Nat. Rev. Drug Discov. 7, 771-782 (2008). 
32. Rees, D. C., Williams, T. N. \& Gladwin, M. T. Sickle-cell disease. Lancet 376, 2018-2031 (2018).

\section{Methods}

Gray scale e-beam lithography. PMMA $950 \mathrm{~K} 11 \%$ in anisol was spin-coated on a 4" Si-wafer at $1000 \mathrm{rpm}$ for $60 \mathrm{~s}$ and baked on a hotplate at $175^{\circ} \mathrm{C}$ for $25 \mathrm{~min}$ to achieve an average film thickness of $4 \mu \mathrm{m}$. The resist contrast was determined by exposing squares of $500 \mu \mathrm{m} \times 500 \mu \mathrm{m}$ with e-beam doses ranging from 0 to $400 \mu \mathrm{C} / \mathrm{cm}^{2}$ in $20 \mu \mathrm{C} / \mathrm{cm}^{2}$ increments by using Raith EBPG $5000+$ electron beam system operated at a $100 \mathrm{kV}$ acceleration voltage. The PMMA 950K was developed in pure methyl isobutyl ketone (MIBK, Technic France) at $20^{\circ} \mathrm{C} 72 \mathrm{~h}$ after exposure, rinsed in isopropanol (IPA, Technic France) for $20 \mathrm{~s}$ and blow-dried with nitrogen. The remaining resist depth of the exposed areas was measured with profilometer (Veeco Dektak 8) using a $2.5 \mu \mathrm{m}$ stylus and plotted against the respective exposure dose to obtain the contrast curve. The TRACER program (GenISys $\mathrm{GmbH}$ ) was used to perform a MonteCarlo simulation of the interaction between the incident electron beam and the resist material to obtain the point-spread function (PSF) at a given penetration depth. The nanofluidic device was designed using a custom developed Python script in a GDSIl format, where each layer was correlated with a different height to achieve the desired 3D topography in the trapping region and the connecting wedge. The contrast curve, PSF and nanofluidic design were loaded into the BEAMER software (GenISys $\mathrm{GmbH}$ ) to perform a 3D proximity effect correction (3D PEC) to ensure a proper dose modulation during subsequent e-beam exposure. An array of 6 devices consisting of three channels was exposed with a base dose of 80 $\mu \mathrm{C} / \mathrm{cm}^{2}$, after which the resist was developed as previously described.

Nanofluidic master fabrication and nanoimprint lithography. A 4" borosilicate wafer was cleaned with acetone and subsequently in IPA, blow-dried with nitrogen and activated with an oxygen plasma (RIE80, Oxford Instruments) at 40W with a pressure of $20 \mathrm{mTorr}$ for $1 \mathrm{~min}$. The adhesion promoter Ormoprime (Microresist) was spin-coated on the activated surface at $4500 \mathrm{rpm}$ for $45 \mathrm{~s}$ and baked on a hotplate at $150{ }^{\circ} \mathrm{C}$ for $5 \mathrm{~min} .1 \mathrm{~mL}$ of the UV crosslinking polymer GMN-PS90 (Optool) was pipetted directly on the surface of the developed e-beam structure, on top of which the borosilicate wafer was placed. The GMNPS90 was allowed to spread for $20 \mathrm{~min}$ to ensure that all the nanofluidic cavities were filled.

Subsequently, the polymer was cured under UV-light at $365 \mathrm{~nm}$ for $6 \mathrm{~min}$ at a power of $300 \mathrm{~mW} / \mathrm{cm}^{2}$. As the GMN-PS90 polymer is inherently anti-sticking, the cured polymer can easily be demolded from the ebeam structures and was immediately ready for further downstream fabrication steps. More specifically, this Optool nanofluidic master was put into a Jenoptik Hex 03 imprinting tool with a $1 \mathrm{~mm}$ freestanding PMMA film on top of it. To ensure a homogeneous imprinting, an anti-adhesion coated silicon wafer was placed on the backside of the PMMA film, with a poly(amide)-PDMS-poly(amide) sandwich to equalize the imprinting pressure. A touch force of $300 \mathrm{~N}$ was applied and the imprinting chamber was steadily heated to a temperature of $130^{\circ} \mathrm{C}$ at $9 \%$ min before the force increased to $17500 \mathrm{~N}$, where it was kept for $15 \mathrm{~min}$. The chamber was then cooled to $60^{\circ} \mathrm{C}$ within 15 minutes before opening and demolding the imprinted PMMA film from the GMN-PS90 master. The free-standing PMMA film was activated by oxygen plasma at $80 \mathrm{~W}$ and a pressure of $0.8 \mathrm{mbar}$ for $20 \mathrm{~s}$ and spin-coated with a protective layer of $10 \%$ 
Dextran (66 kDa, Roth Industries) solution in deionized water at $3000 \mathrm{rpm}$ for $60 \mathrm{~s}$. The array of 6 devices was then mechanically cut into two separate $3 \times 1$ devices and the particle protecting Dextran layer was removed by submerging the chips in MilliQ water for 10 min and subsequent drying in a vacuum chamber at 9 mbar for $10 \mathrm{~min}$.

Poly(vinyl alcohol) (PVA) functionalization and bonding. The PMMA surfaces of the $1 \mathrm{~mm}$ patterned film and a $200 \mu \mathrm{m}$ unpatterned film were activated by UV/O-activation at $172 \mathrm{~nm}$ for $30 \mathrm{~s}$. This reduces the molecular weight of surface polymer chains and consequently also the surface glass transition temperature ( $T_{g}$, surface $)$ with respect to the bulk material. This leads to increased interactions of PMMA chains and the surface contact area, much like in a nanovelcro-like system. Subsequently, a 0.5\% PVA (Polysciences, 15132-500) solution in PBS pH 7.4 was spin-coated at 2000 rpm for 1 min. To remove any excess PVA and aid film homogeneity, the surface of the PMMA nanofluidic chip was spin-washed with deionized water at 2000 rpm for $1 \mathrm{~min}$. Afterward, the patterned and unpatterned PMMA films were aligned and bonded at $750 \mathrm{~N}$ and $45^{\circ} \mathrm{C}$ for $1 \mathrm{~min}$.

Trapping of calibration grade fluorescent polystyrene particles. A five-particle mixture in PBS was made with calibration grade fluorescent polystyrene particles of the following sizes and dyes: $0.9 \mu \mathrm{m}$ (EstaporX,

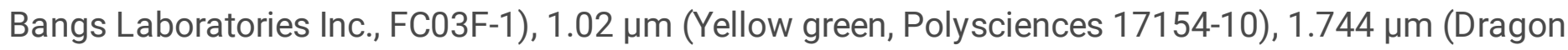
Green, Polysciences 683195), 2.12 um (Polychromatic red, Polysciences 19508-2), $3.16 \mu \mathrm{m}$ (Yellow green, Polysciences 18861-1). A $4 \mu \mathrm{l}$ droplet was put onto the inlet of the nanofluidic device and after the liquid reached the end of the capillary pump region, a droplet of PBS was placed at the outflow to prevent premature drying of the sample. The fluorescence microscopy was done with a Leica SP8 microscope using a 40x objective with 0.95 numerical aperture. A custom python script was used to analyze the average trapping positions of the particles and corresponding standard deviation. To compensate for the lateral height discrepancy in the channel, only the trapping in the central region of the channel was considered for the calculation of the trapping position. The particle sizes specified by the supplier were used to fit a linear regression curve to the particle trapping position. The mean particle size and coefficient of variation were recalculated from the linear regression fit.

Fluorescence microscopy. The trapped particles in the active region of the nanofluidic device were fluorescently investigated with a Leica DMi8 microscope using a 10x objective with a 0.32 numerical aperture. The microscope is equipped with an LED light source with a tunable excitation wavelength and a Leica-K5-14400713 detector. To ensure proper filtration of the emission light, filter cubes for the following wavelengths were used: 700, 585, 527, $470 \mathrm{~nm}$. The same setup was employed when examining the filling of the device's capillary pump region with ATTO 448. For the on-chip SARS-CoV-2 and Influenza A antibody assays a 40x objective with a 0.95 numerical aperture was used.

Preparation of Dynabeads M-280 and MyOne T1 with spike-RBD and hemagglutinin. Dynabeads M-280 (Thermo Fischer Scientific, 11205D) were added to $1 \mathrm{~mL}$ of PBS and left on the Spin Magnet (Thermo Fischer Scientific, 12320D) for 4 min to ensure proper magnetic isolation of the beads. Afterward, the beads were washed twice with the same volume of PBS before suspending the washed beads in the final 
volume used for labeling. These suspensions were mixed together with the biotinylated receptor-binding domain (RBD) spike protein (Sino Biological, 40592-V08B-B) at $600 \mathrm{rpm}$ and $25^{\circ} \mathrm{C}$ for $2 \mathrm{~h}$, with resuspending the mixture every $30 \mathrm{~min}$. Subsequently, the beads were washed three times in PBS as previously described. For the COVID-19 only experiments, the particles were blocked with Superblock PBS (Thermo Fischer Scientific, 37580) for $30 \mathrm{~min}$ at $600 \mathrm{rpm}$ and $25^{\circ} \mathrm{C}$, whereas for the influenza multiplexing experiments, $0.9 \mathrm{mg} / \mathrm{mL}$ free biotin (Thermo Fischer Scientific, 29129) was added during the blocking step to saturate all remaining open streptavidin binding pockets. Dynabeads MyOne T1 (Thermo Fischer Scientific, 65601) were washed as described above and were labeled with biotinylated Influenza A H1N1 Hemagglutinin (A/California/04/2009, Sino Biological, 11055-RP01). The beads were subsequently blocked in Superblock PBS containing $0.9 \mathrm{mg} / \mathrm{mL}$ free biotin for $30 \mathrm{~min}$ at $600 \mathrm{rpm}$ and $25^{\circ} \mathrm{C}$.

Serological immunoassay - limit-of-detection and test validation. The proof-of-principle of on-bead and on-chip immunoassay was performed by using a chimeric antibody (Sino Biological, 40150-D001) and diluting it in COVID-19 negative human serum (Sigma-Aldrich, H4522). The serum was diluted 1:4 with a spike RBD-functionalized Dynabead 280 suspension and left to incubate at $600 \mathrm{rpm}$ for $1 \mathrm{~h}$ at $25^{\circ} \mathrm{C}$ ) after which it was washed three times with PBS (pH 7.4). To validate the binding of the anti-S-RBD antibodies to the antigen-coated beads, the washed particles were incubated with Cy 5 donkey anti-human IgG, diluted according to the supplier's recommendations. This was done for $1 \mathrm{~h}$ at $25^{\circ} \mathrm{C}$ and $600 \mathrm{rpm}$, with three subsequent washing steps. To control the flow and trapping functionality of the device, $0.9 \mu \mathrm{m}$ non-magnetic Estaporix-loaded polystyrene beads were added to the suspension. A $4 \mu \mathrm{l}$ droplet of the suspension was then loaded onto the 3D nanofluidic PMMA chip and after the liquid reached the end of the capillary pump, a droplet of PBS ( $\mathrm{pH}$ 7.4) was pipetted on the outflow region to halt the further fluid flow. The fluorescent signal in the relevant trapping region was quantified by using a custom Python image analysis script. The script used the scikit-image module to identify the relevant fluorescent pixels, perform the background subtraction and the equalization with a top-hat filter. Afterward, NumPy and Pandas were used to calculate the mean signal of the acquired image. Assay Validation. The assay described above was performed with a 29-patient sample set (Anawa GmbH, COV-POSSET-S), consisting of $19 \mathrm{lgG}$ positive and $10 \mathrm{lgG}$ negative patients. All of the IgG-positive patients had a positive PCR test for COVID-19, whereas the IgG-negative patients had a negative PCR test. The highest fluorescence signal of the negatively-tested patient population was taken as a cut-off value at 6.159 arbitrary units. A higher signal was considered to be a positive result.

Antibody and disease multiplexing. Antibody multiplexing. Dynabeads M-280 were labeled with the COVID-19 spike RBD as previously outlined. Afterward, they were mixed in a 3:4 ratio and incubated with serum from a positive patient control with a high ELISA titer for IgG and IgM antibodies against the spike RBD as well as with a negative patient control, which was tested PCR negative for COVID-19. To achieve color-multiplexed antibody detection, two secondary antibodies were added to the particle suspension: a donkey anti-human IgM Alexa 488 (Bioconcept, 2020-30) and donkey anti-human IgG Cy5 (Jackson Immuno, AB_2340539). Both were incubated with the washed particle suspension as described in the previous section. The device loading and investigation as well as associated analysis, were performed in the same fashion. Disease multiplexing. As a proof-of-principle, a particle suspension containing spike 
RBD functionalized Dynabeads M-280 and hemagglutinin functionalized Dynabeads MyOne T1 particles were mixed with purified polyclonal rabbit anti-spike (Sino Biological, 40592-T62) and purified polyclonal rabbit anti-hemagglutinin (Sino biological, 11055-RP01). The beads were then immunostained with donkey anti-rabbit Cy3 (Jackson Immuno, AB_2307443) for $1 \mathrm{~h}$ at $25^{\circ} \mathrm{C}$ and $600 \mathrm{rpm}$ after which the suspension was washed three times with PBS ( $\mathrm{pH}$ 7.4). $0.9 \mu \mathrm{m}$ Estaporix flow control beads were added before loading the particles into the nanofluidic device. The relevant trapping lines were investigated and analyzed as previously described.

\section{Figures}

(a)

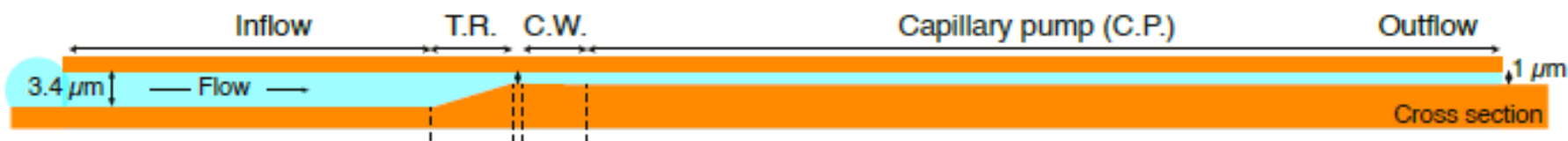

(b)

(c)

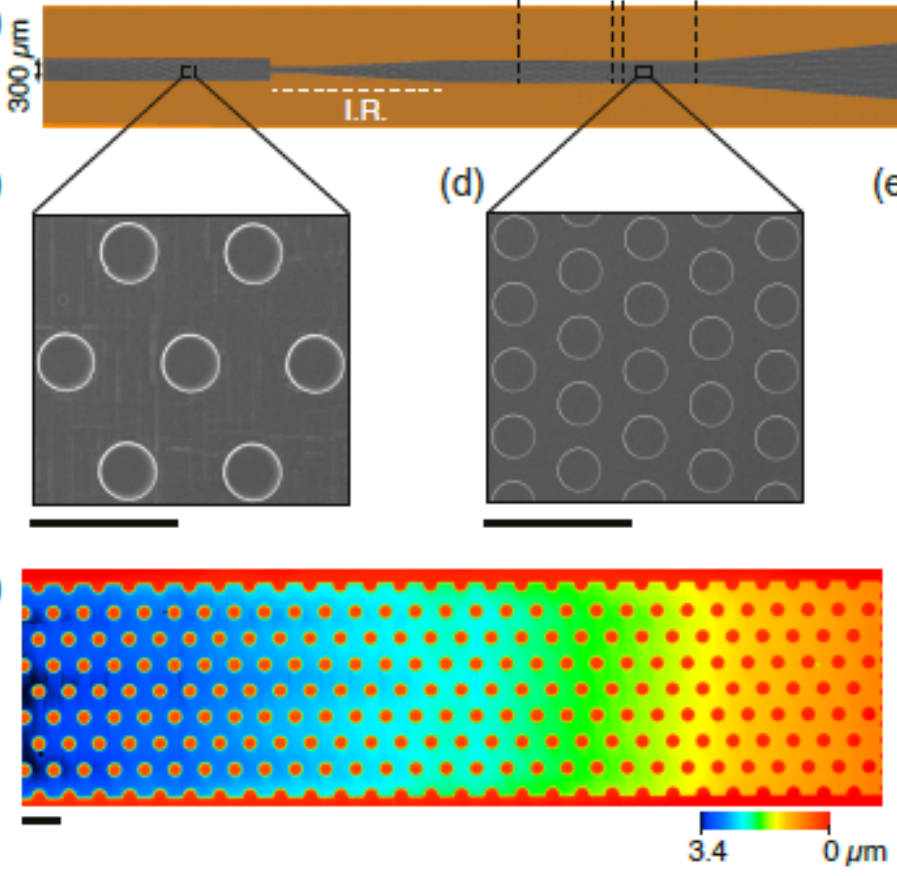

(h)

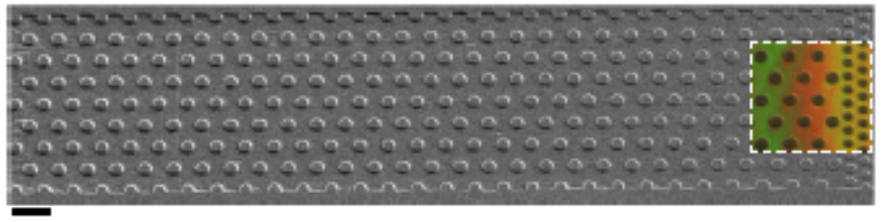

(i) Filling front

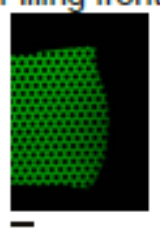

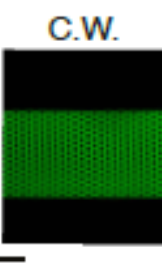

Capillary pump

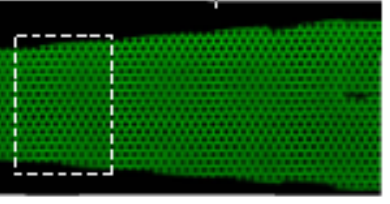

(e)
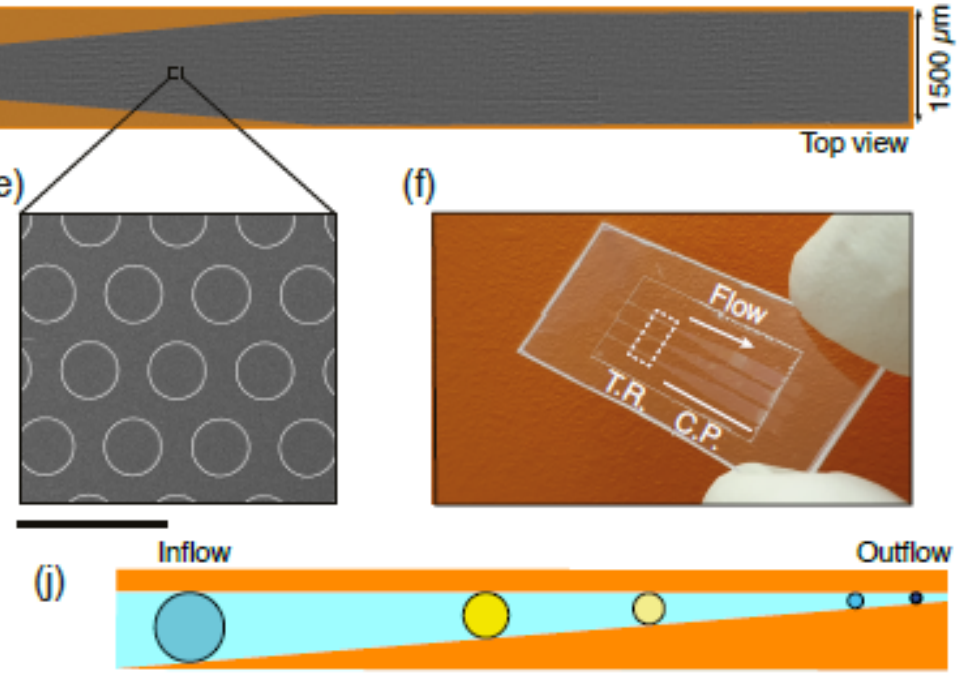

(k)
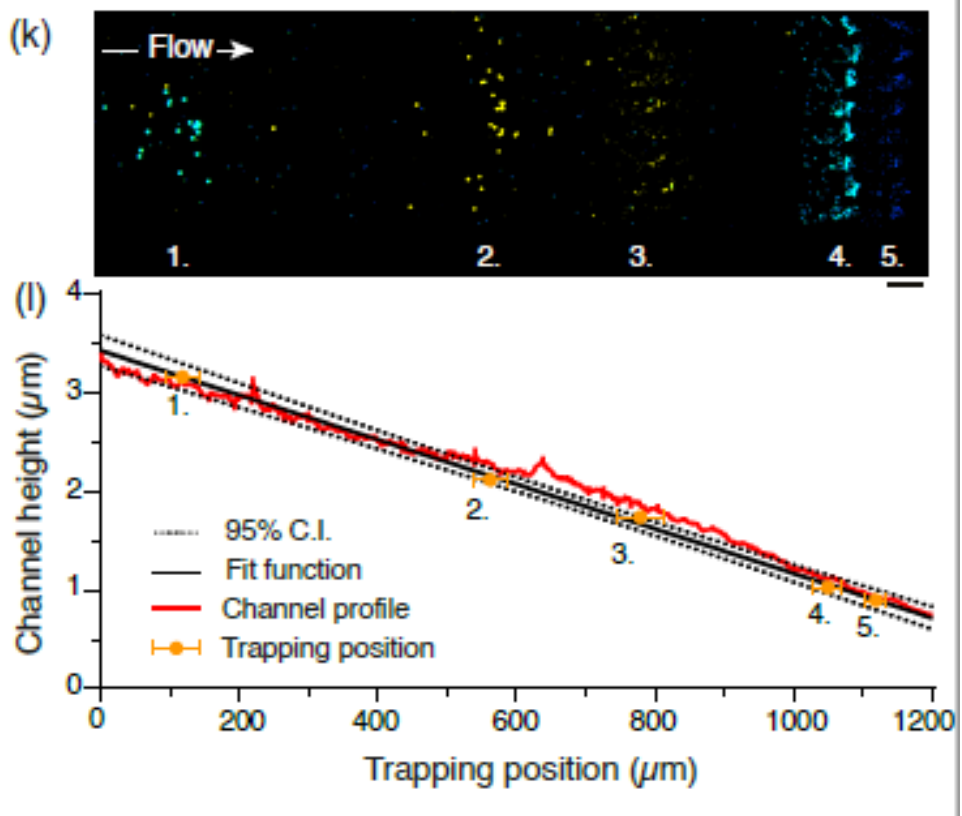

Figure 1 
3D nanofluidic PMMA device. a, Cross-sectional view of the changing height profile inside the device $b$, Scanning Electron Microscopy (SEM) top view image of the device, with the inflow resistor (I.R.) marked by a white dashed line c-e, SEM images of supporting pillars with a diameter of $20 \mu \mathrm{m}(\mathrm{c}, \mathrm{f})$ and $15 \mu \mathrm{m}(\mathrm{e})$ in the different regions of the device $f$, photograph of the 3D PMMA nanofluidic device showing the narrower inflow and broader capillary pump region. Each device contains three channels which have the same geometrical layout to allow for multiple measurements of the same sample on one device $\mathrm{g}$, topographical confocal laser scanning image of the trapping region showing the decreasing channel height over the channel length $\mathrm{h}$, SEM image of the trapping region recorded at a $30^{\circ}$ tilt-angle with an inset of a light microscopy image of a bonded device at the transition between the trapping and connecting region $\mathrm{i}$, Fluorescent image of the filling of the connecting wedge and capillary pump region with ATTO488 and the associated filling front as indicated by the white dashed square j, Cross-sectional sketch illustrating the immobilization of calibration grade particles of different sizes and colors $k$, Fluorescent micrograph of trapped fluorescent polystyrene calibration grade particles I, Plot correlating the mean trapping position with the mean particle size. The error bars represent the standard deviation of the trapping position. The dotted lines show the $95 \%$ confidence interval of the linear regression fit. All scalebars represent a $50 \mu \mathrm{m}$ length. 
(a)

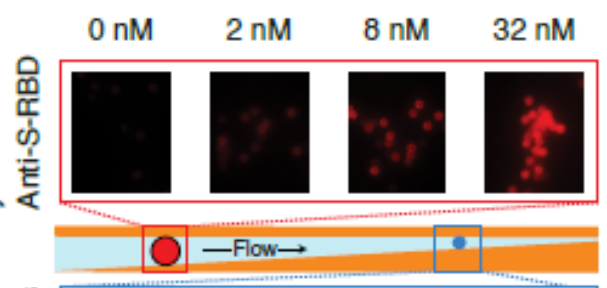

-

(c)

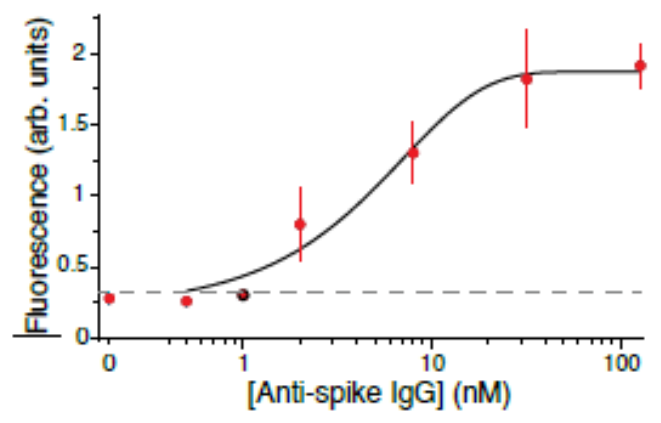

(e)

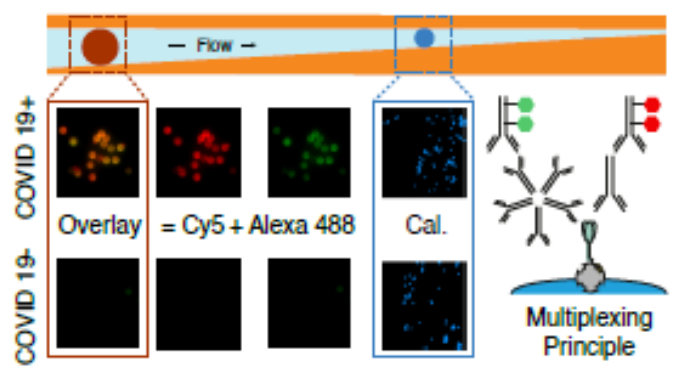

(g)

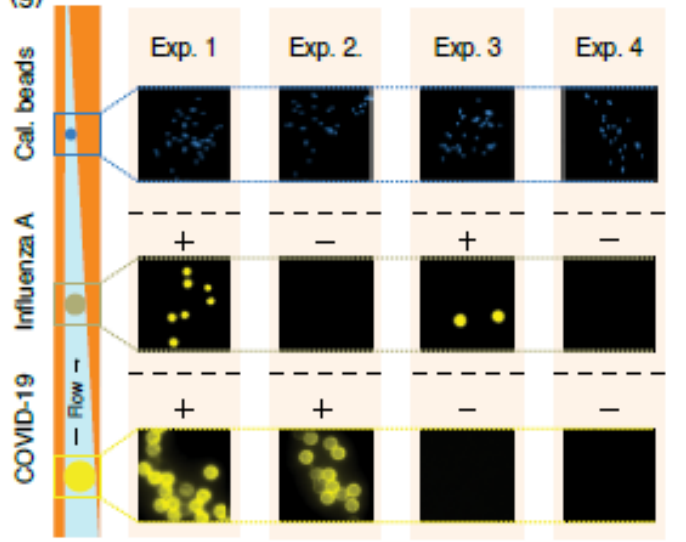

(b)

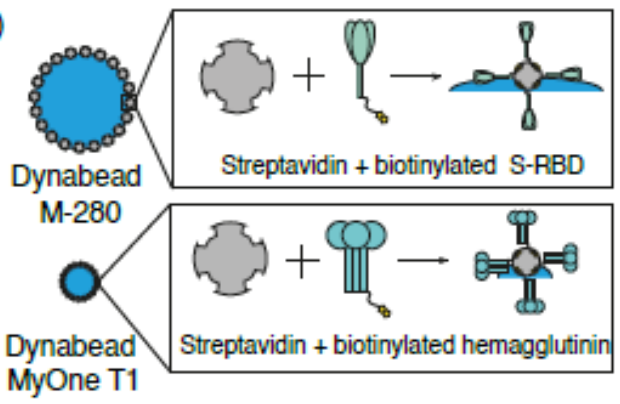

(d)

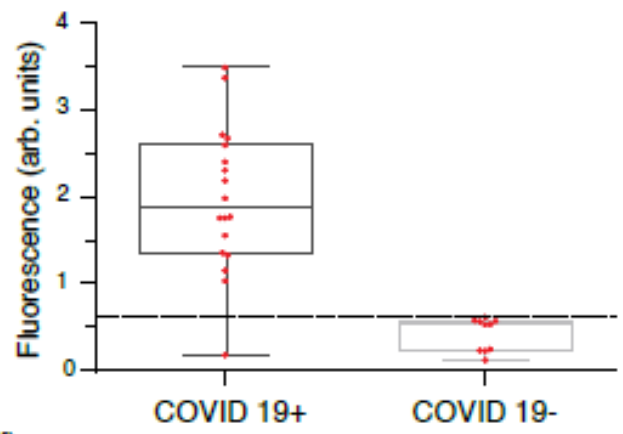

(f)

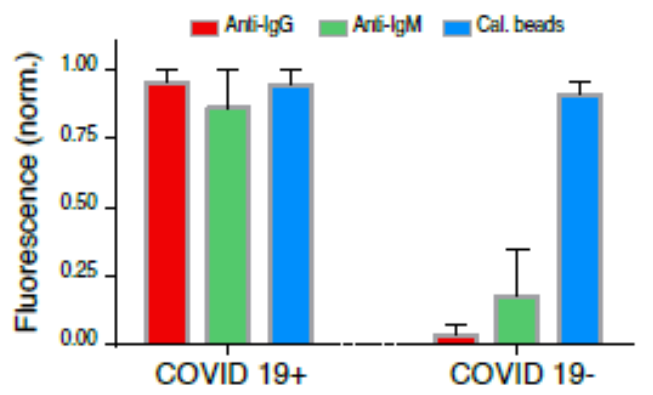

(h)

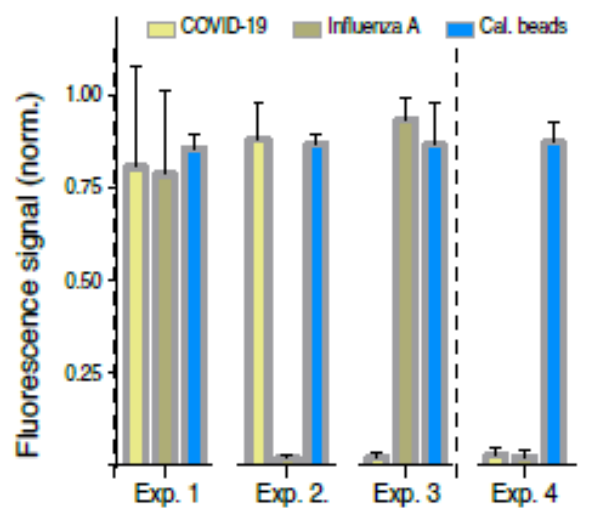

Figure 2

On-chip COVID-19 and Influenza A antibody immunoassay. a, Top: Fluorescence images of trapped Dynabeads M-280 mixed with a given concentration of anti-S-RBD antibodies in human serum. Middle: cross-section of the trapping region. Bottom: Trapped $0.9 \mu \mathrm{m}$ EstaporX flow control beads $b$, Functionalization of streptavidin-coated Dynabeads M-280 the receptor-binding domain (RBD) of the spike protein. For simplicity, the entire trimeric protein is drawn with a single biotin moiety. The smaller 
Dynabeads MyOne T1 beads (bottom) are similarly functionalized with a biotinylated hemagglutinin protein c, quantification of anti-human IgG Cy5 fluorescent signal on trapped Dynabeads M-280 for a given anti-spike IgG concentration. The dashed line represents the estimated LOD d, Validation of on-chip anti-spike IgG immunoassay using COVID-19 PCR positive and negative patient samples. Each data point represents the mean signal of each patient. The box plot has whiskers from the minimum to maximum value, whereas the dashed line graphically indicates the cut-off value separating negative from positive values e, Top: schematic of a color multiplexed IgM and IgG on-bead immunoassay. Bottom: the resulting fluorescent signal resulting from the on-chip immunoassay for a COVID-19 positive and - patient $f$, Normalized signal of anti-human IgG cy5, anti-human IgM Alexa 488 and calibration bead fluorescent signal g, Left: Schematic of a multiplexed on-chip immunoassay for simultaneous detection of anti-spike (SARS-CoV-2) and anti-hemagglutinin H1N1 (Influenza A) antibodies. Right: fluorescent micrograph of trapped particles of different sizes and different antigen functionalities $h$, Normalized anti-rabbit Cy3 signal at the trapping lines for Dynabeads M-280 and MyOne T1, testing for the presence of SARS-CoV-2 and Influenza A antibodies, respectively.

\section{Supplementary Files}

This is a list of supplementary files associated with this preprint. Click to download.

- SupplementaryFigure1.pdf

- SupplementaryFigure2.pdf

- SupplementaryFigure3.pdf

- SupplementaryFigure4.pdf

- SupplementaryFigure5.pdf

- Supportingvideo1.mp4

- Supportingvideo2.mp4

- SupplementaryTable1.docx 